\title{
All-optical linear polarization engineering in single and coupled exciton-polariton condensates
}

\author{
I. Gnusov, ${ }^{1, *}$ H. Sigurdsson, ${ }^{2,3, \dagger}$ J. D. Töpfer, ${ }^{1}$ S. Baryshev, ${ }^{1}$ S. Alyatkin,${ }^{1}$ and P. G. Lagoudakis ${ }^{1,3}$ \\ ${ }^{1}$ Skolkovo Institute of Science and Technology, Moscow, \\ Territory of innovation center "Skolkovo", Bolshoy Boulevard 30, bld. 1, 121205, Russia. \\ ${ }^{2}$ Science Institute, University of Iceland, Dunhagi 3, IS-10\%, Reykjavik, Iceland \\ ${ }^{3}$ School of Physics and Astronomy, University of Southampton, Southampton, SO17 1BJ, UK.
}

\begin{abstract}
We demonstrate all-optical linear polarization control in semiconductor microcavities using an exciton-polariton condensate in an elliptically shaped optical trap. The microcavity inherent TETM splitting lifts the pseudospin degeneracy of the anisotropic trap ground state. The emerging fine structure modes are shown to be polarized linearly parallel and perpendicular to the trap major axis. We demonstrate polariton condensation into the excited pseudospin mode with high degree of linear polarization which rotates as we rotate the trap. We then extend our study to a system of two coupled linearly polarized condensates and demonstrate rich spin dynamics reflecting spontaneous synchronization and high correlation between the condensate pseudospins as a function of pump parameters. Our findings show a new type of polarization control with exciting perspectives in both spinoptronics and studies on extended systems of interacting nonlinear optical elements with anisotropic coupling strength and adjustable fine structure.
\end{abstract}

\section{INTRODUCTION}

Exciton-polaritons (polaritons hereafter), often referred to as liquid light, are a mixture of both light and matter. They arise in the strong coupling regime between quantum well excitons and cavity photons in semiconductor microcavities ${ }^{1}$. Being composite bosons, they can undergo a power-driven nonequilibrium phase transition into a highly coherent many-body state referred as a polariton condensate ${ }^{2}$. An essential characteristic of these liquid light particles is their pseudospin (or just spin) projection $( \pm \hbar)$ onto the growth axis of the cavity which can easily be determined by measuring the polarization of the polariton photoluminescence (PL).

The strong nonlinear nature of polaritons through their spin-anisotropic excitonic Coulomb interactions results in numerous intriguing spinor condensate properties desirable in future spinoptronic technologies ${ }^{3,4}$. This includes spin bistability ${ }^{5-7}$ and multistability ${ }^{8}$, switches ${ }^{9,10}$, optical spin Hall effect ${ }^{11}$, polarized solitons ${ }^{12,13}$ and vortices $^{14-16}$, spin bifurcation points ${ }^{17}$, and topological phases $^{18,19}$. Different parts for polariton based spin circuitry have already been realized ${ }^{9,10,20-22}$ with some recent exciting theoretical proposals ${ }^{23,24}$, but many challenges remain to be solved. Optical applications such as data communication or sensing benefit from precise control over a laser's polarization and modulation speeds, ideally using nonresonant excitation schemes like spinVCSELs $^{25-27}$. In this spirit, a great deal of effort has been devoted to generating sources of linearly polarized light such as colloidal nanorods ${ }^{28}$, materials with anisotropic optical properties ${ }^{29}$, quantum $\operatorname{dots}^{30}$, and optical parametric oscillators ${ }^{31}$.

Under nonresonant excitation in inorganic semiconductors, spin transfer from the pumping laser to the condensate is possible by using an elliptically polarized beam which creates a spin-imbalanced polariton gain (i.e., op- tical orientation of excitons $)^{6,32}$. This allows generating condensates of high degree of circular polarization aligned with the pump. However, the linearly polarized polariton modes experience isotropic gain, making them insensitive to the linear polarization details of the nonresonant excitation source ${ }^{33,34}$ except in the presence of cavity strain and birefringence ${ }^{32,35-39}$ or engineered anisotropic cavities ${ }^{40,41}$. The same also applies for VCSELs, where the linear polarization of the emission is engineered by etching asymmetric masks ${ }^{42,43}$ or electrodes $^{44}$, heating ${ }^{45}$, or applying mechanical stress ${ }^{26}$. Alternatively, in organic polaritonics, single-molecule Frenkel excitons can be excited by a linearly polarized nonresonant pump co-aligned with their dipole moment leading to polariton condensation of the same linear polarization ${ }^{46}$. However, control over both circular and linear polarization degrees of freedom in a polariton condensate through nonresonant all-optical means has remained elusive.

Here, we demonstrate in-situ optical control of the linear polarization in inorganic polariton condensates in a cavity with polarization-dependent reflectivity, or TETM splitting ${ }^{11,47}$. By spatially shaping the nonresonant excitation laser profile into the form of an ellipse, we are able to fully control the direction of the condensate linear polarization. Our elliptically shaped pump induces an anisotropic in-plane trapping potential and gain media for the polaritons. Such an excitation profile along with the cavity TE-TM splitting leads to condensation (lasing) into a mode of definite linear polarization parallel to the minor axis of the trap ellipse. The optical malleability of the trap geometry allows for non-invasive deterministic control over the linear polarization of the condensate by just utilizing the nonresonant excitation laser. Moreover, we investigate the effects of anisotropic coupling between two spatially separated condensates and identify several different regimes of exotic linear polarization dynamics. 


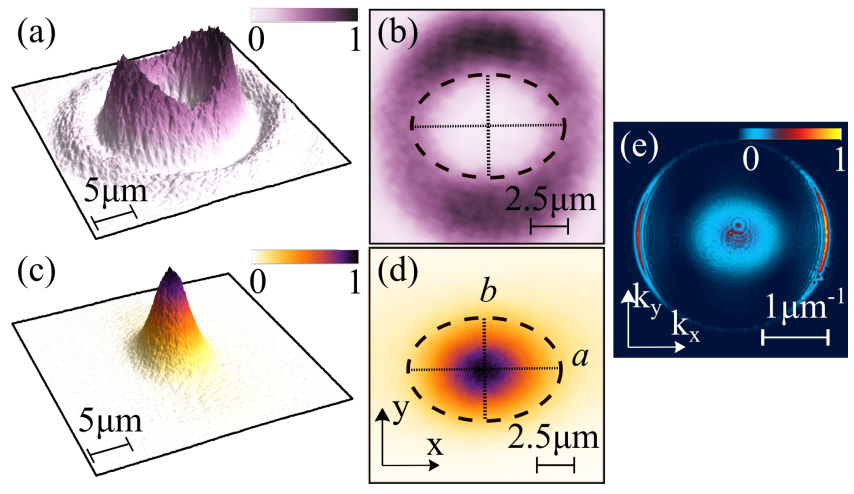

Figure 1. Spatial intensity profiles of the $(\mathrm{a}, \mathrm{b})$ excitation laser and $(\mathrm{c}, \mathrm{d})$ condensate PL. The excitation laser induces a trapping potential with horizontal and vertical radii denoted $a, b$ respectively. (e) Momentum distribution of the condensate PL. Panels $(\mathrm{c}, \mathrm{d}, \mathrm{e})$ correspond to a condensate pumped twice above its condensation threshold (i.e., $P=2 P_{t h}$ ).

\section{EXPERIMENT}

Our experiments are conducted on an inorganic $2 \lambda$ $\mathrm{GaAs} / \mathrm{AlAs}_{0.98} \mathrm{P}_{0.02}$ microcavity with embedded InGaAs quantum wells ${ }^{48}$. The sample is excited nonresonantly by a linearly polarized continuous wave $(\mathrm{CW})$ laser $(\lambda=$ $783.6 \mathrm{~nm}$ ). The optical excitation beam is chopped using an acousto-optic modulator to form $10 \mu$ s square pulses at $1 \mathrm{kHz}$ repetition rate to diminish heating of the sample held at a temperature of $4 \mathrm{~K}$. The exciton-cavity mode detuning is $-3 \mathrm{meV}$. A reflective, liquid-crystal spatial light modulator (SLM), commonly used today for optical shaping of polariton condensates, transforms the transverse profile of the pump laser beam to have an elliptically shaped confinement region [see Fig. 1(a) and dashed ellipse in Fig. 1(b)]. We investigate the sample PL in real [Fig. 1(c,d)] and reciprocal [Fig. 1(e)] space, and record the time- and space-averaged polarization of the PL by simultaneously detecting all polarization components ${ }^{32}$.

The polariton condensate order parameter is written in the canonical spin-up and spin-down basis $\Psi=$ $\left(\psi_{+}, \psi_{-}\right)^{T}$ corresponding to left- and right-circularly polarized condensate emission, respectively. It is convenient to use the pseudospin formalism corresponding to the Stokes vector of the emitted light $\mathbf{S}=\left(S_{1}, S_{2}, S_{3}\right)^{T}=$ $\left\langle\Psi^{\dagger} \hat{\boldsymbol{\sigma}} \Psi\right\rangle /\left\langle\Psi^{\dagger} \Psi\right\rangle$ where $\hat{\boldsymbol{\sigma}}$ is the Pauli matrix vector. The polariton PL is analyzed in terms of time-averaged Stokes components which are written as,

$$
S_{1}=\frac{I_{H}-I_{V}}{I_{H}+I_{V}}, S_{2}=\frac{I_{D}-I_{A}}{I_{D}+I_{A}}, S_{3}=\frac{I_{\sigma^{+}}-I_{\sigma^{-}}}{I_{\sigma^{+}}+I_{\sigma^{-}}},
$$

where $I_{H, V, D, A, \sigma^{+}, \sigma^{-}}$are the time-averaged intensities of horizontal, vertical, diagonal, antidiagonal, right- and left circular polarization projections of the emitted light respectively.

We start by exciting the sample with a symmetric ring-shaped pump profile [see inset in Fig. 2(a)], creat-

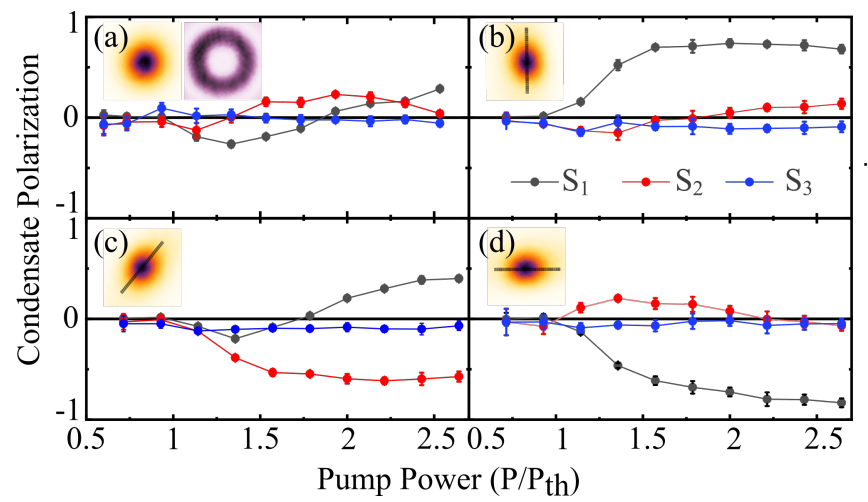

Figure 2. (a) Power dependence of the condensate $S_{1,2,3}$ Stokes parameters (black, red, blue markers) for the annular pump with resultant cyllindrically symmetric condensate profile (see right and left insets, respectively). (b) Same but now for a trap/condensate with a major axis orientated at $90^{\circ}$, (c) $45^{\circ}$, and (d) $0^{\circ}$. Insets in (b)-(d) depict the realspace condensate PL with black lines showing the orientation of the trap major axis.

ing a two-dimensional trap for the polaritons. Ramping the pump power above the condensation threshold denoted as $P_{t h}$ we obtain condensation into the fundamental trap mode. The optical trap is realized by the strong polariton repulsive interactions with the background pump-induced cloud of incoherent excitons. This reservoir of excitons forms a blueshifting potential onto the polaritons ${ }^{49,50}$, while at the same time providing gain. Such an optical trapping technique has the advantage of reducing the overlap between the condensate and the exciton reservoir, minimizing detrimental dephasing effects $^{51}$. By scanning the excitation position with the ring-shaped pump profile we locate a spot on our sample with small degree of polarization DOP $=\sqrt{S_{1}^{2}+S_{2}^{2}+S_{3}^{2}}$ [see Fig. 2(a)] of the emitted light. The small $S_{1,2}$ above threshold implies that the trap ground state is spindegenerate such that from realization to realization random linear polarization builds up which averages out over many shots. Small $S_{3}$ confirms that our laser is (to a good degree) linearly polarized and doesn't break the spin parity symmetry of the system. Effects of using elliptically polarized pumps are discussed in Sec. S1 in the Supplemental Information (SI). We also note that in our presented data we have compensated for the effects of optical retardance arising in our setup (see Sec. S2 in SI for further details).

\section{CONDENSATE LINEAR POLARIZATION CONTROL}

\section{A. Rotation of the elliptical trap}

We then transform the excitation profile to the one shown in Figs. 1(a) and 1(b). Non-uniform distribution of the intensity in the excitation leads to the formation of 


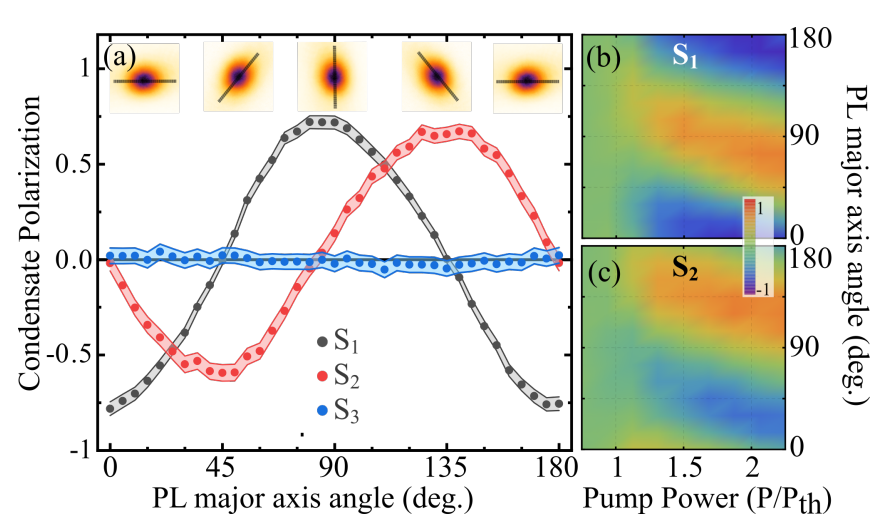

Figure 3. (a) Condensate Stokes parameters for different orientations of the condensate major axis in real space (insets) at $P=1.94 P_{t h}$. Black lines depict the major axis of the trap. Colored regions show error of the measurement. (b) $S_{1}$ and (c) $S_{2}$ colormaps for varying pump powers and major axis orientation showing counter-clockwise rotation of the pseudospin with increasing power.

an elliptically shaped optical trap denoted by the dashed ellipse, squeezing the condensate as shown in Fig. 1(d). We now observe a massive increase of the condensate's linear polarization components above $1.2 P_{t h}$ at the same sample position. The direction of the linear polarization of the emission is found to follow the trap minor axis. Namely, for the vertically elongated condensate in Fig. 2(b) we observe an increase of the $S_{1}$ Stokes component (horizontal polarization). The same effect is present for the diagonally and horizontally elongated condensates in Figs. 2(c) and 2(d). We note that the results are independent on the angle of linear polarization of the pump laser [see Sec. S1 in the SI].

By rotating the excitation profile with the SLM, we can engineer any desired linear polarization in the condensate. In Fig. 3(a), we present the measured polarization components of the condensate as a function of the condensate major axis angle. We observe a continuous rotation of the condensate polarization close to the equatorial plane of the Poincaré sphere following the minor axis of the trap. We point out that DOP $<1$ appears from various depolarizing effects such as noise due to scattering from the incoherent reservoir to the condensate ${ }^{39}$, polariton-polariton interactions in the condensate causing self-induced Larmor precessions ${ }^{52}$, and mode competition $^{53}$.

We have also checked that the results of our study are not dependent on the precise shape of our optical trap. In Sec. S3 in the SI we have tested another geometrical construction of the excitation profile in the form of 8 Gaussian beams arranged to form an ellipse and retrieve qualitatively the same result as presented here in the main text.

Figures 3(b) and 3(c) show pump power and trap orientation dependence of the $S_{1,2}$ Stokes parameters. Interestingly, with increasing pump power we observe counterclockwise rotation of the pseudospin in the equatorial plane of the Poincaré sphere. The rotation is approximately $30^{\circ}$ between 1.2 and $2.2 P_{t h}$. This effect appears due to a small amount of circular polarization in our pump which creates a spin-imbalanced trapping potential and gain media which acts as a complex populationdependent out-of-plane magnetic field $\boldsymbol{\Omega}_{\perp}$ that applies torque on the condensate pseudospin. This is confirmed through simulations using the generalised GrossPitaevskii equation [see Sec. S10 in the SI]. Further analysis on this power dependent trend of the $S_{1,2}$ is beyond the scope of the current study.

\section{B. Theory of a single condensate in an elliptically shaped optical trap}

Our observations can be interpreted in terms of photonic TE-TM splitting acting on the optically confined polaritons which, when the trap $V(\mathbf{r})$ has broken cylindrical symmetry, leads to fine structure splitting in the trap transverse modes. This determines a state of definite polarization which the polaritons condense into. In the non-interacting regime polaritons obey the following Hamiltonian,

$$
\hat{H}=\frac{\hbar^{2} k^{2}}{2 m}-\hat{\boldsymbol{\sigma}} \cdot \boldsymbol{\Omega}+V(\mathbf{r})-\frac{i \hbar \Gamma}{2},
$$

where $m$ is the effective mass, $\mathbf{k}=\left(k_{x}, k_{y}\right)$ is the in-plane cavity momentum, $\Gamma^{-1}$ is the polariton lifetime, and

$$
\boldsymbol{\Omega}=\hbar^{2} \Delta\left(\begin{array}{c}
k_{x}^{2}-k_{y}^{2} \\
2 k_{x} k_{y} \\
0
\end{array}\right)
$$

is the effective magnetic field [see Fig. 4(a)] from the TE-TM splitting of strength $\Delta^{11}$ acting on the polariton pseudospin. Note that the minus sign in front of the magnetic potential energy is conventional but many other works use a positive sign with no loss of generality. For elliptical confinement, which we assume to be harmonic for simplicity $V(\mathbf{r})=m\left(\omega_{x}^{2} x^{2}+\omega_{y}^{2} y^{2}\right) / 2$, the TE-TM splitting lifts the degeneracy of the trap spin-levels. For the lowest trap state the magnetic field reads (see Sec. S5 in the SI for detailed derivation),

$$
\boldsymbol{\Omega}_{\text {trap }} \simeq-\frac{\hbar m|\Delta| \delta \omega}{2}\left(\begin{array}{c}
\cos \left(2 \theta_{\text {min }}\right) \\
\sin \left(2 \theta_{\text {min }}\right) \\
0
\end{array}\right) .
$$

Here, $\theta_{\min }$ is the angle of the trap minor axis from the horizontal, and $\delta \omega=\left|\omega_{x}-\omega_{y}\right| \propto\left|a^{-1}-b^{-1}\right|$ is the absolute difference between the trap oscillator frequencies along the major and the minor axis [Fig. 1(d)]. We point out that the minus sign in Eq. (4) is written explicitly because $\Delta<0$ in our sample ${ }^{54}$ (see Sec. S4 in the SI).

The direction of the effective magnetic field is controlled by the angle of our elliptical trap, $\theta_{\min }$, which consequently rotates the condensate pseudospin in the 


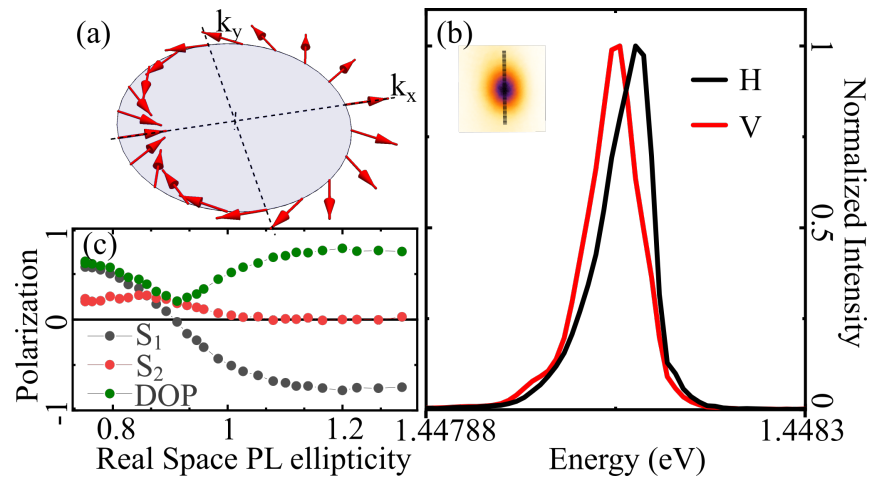

Figure 4. (a) Distribution of the in-plane effective magnetic field $\boldsymbol{\Omega}(\mathbf{k})$ (red arrows) in momentum space due to TE-TM splitting given by Eq. (3). (b) Horizontal (black) and vertical (red) polarization resolved normalized spectrum of the condensate emission at $k=0$ for a vertically elongated trap $\left(\theta_{\min }=0\right)$. Splitting between levels is $\approx 20 \mu \mathrm{eV}$. (c) Linear polarization components and DOP for different real-space ellipticities of the condensate. Data was taken at $P \approx 1.8 P_{t h}$

equatorial plane of the Poincaré sphere such that it stabilizes antiparallel to the magnetic field $-\mathbf{S} \| \boldsymbol{\Omega}_{\text {trap }}$. This leads to smooth changes in the $S_{1,2}$ Stokes components of the emitted light as the trap rotates like shown in Fig. 3. The results of our experiment are accurately reproduced through a mean-field theory using a generalized Gross-Pitaevskii model describing the polariton condensate spinor order parameter $\Psi$ coupled with a background excitonic reservoir (see Sec. S6 in the SI).

Interestingly, in a recent experiment ${ }^{32}$ we observed condensation into the spin ground state of a circular trap, where the fine structure splitting originated from the cavity birefringence $\boldsymbol{\Omega}_{\text {bir }}(\mathbf{r})$. This meant that the condensate pseudospin stabilized parallel to the magnetic field $\mathbf{S} \| \boldsymbol{\Omega}_{\text {bir }}(\mathbf{r})$. In the current experiment however, we instead observe condensation into the excited spin state, i.e. antiparallel to the magnetic field $-\mathbf{S} \| \boldsymbol{\Omega}_{\text {trap }}$. This can be directly evidenced in Fig. 4(b) where we show the normalized polarization-resolved spectrum of a vertically elongated trap which obtains a horizontally polarized condensate that is higher in energy.

Performing linear stability analysis on a GrossPitaevskii mean field model (see Sec. S7 in the SI) we determine that repulsive polariton-polariton interactions normally leads to condensation in the fine structure ground state ${ }^{39}$. However, the additional presence of an uncondensed background of reservoir excitons contributes to an effective attractive mean-field interaction in the condensate ${ }^{55}$ which causes the ground state to become unstable, favouring condensation into the excited state as we observe in the current experiment. Another effect is the different penetration depths of the linearly polarized polariton modes (due to their different effective masses from the TE-TM splitting) into the excess gain region about the trap short axis. This leads to higher gain for the fine structure excited state which facilitates its condensation. Several parameters of the polariton system such as exciton-photon detuning, the quantum well material, and shape of the pump profile allow tuning from one stability regime to another which explains why some experiments show ground-state condensation ${ }^{32}$ while other, like ours, show exited-state condensation ${ }^{56}$. We stress that regardless of whether system parameters favour condensation into the spin ground- or excited state of the optical trap, the main result of our study remains valid.

\section{Changing the trap spatial ellipticity}

So far we have shown control over the linear polarization of the condensate by rotating the trap major axis and varying the pump power. Here, we show that the condensate linear polarization can also be controlled by tuning the trap spatial ellipticity (i.e., ratio of major and minor axis). In Fig. 4(c) we continuously change the trap spatial ellipticity from a vertically elongated trap $(a<b)$ to a horizontally elongated one $(a>b)$. The PL spatial ellipticity axis denotes the ratio of width of the PL along $x$ - and $y$-axis [see Fig. 1(d)]. The pseudospin of the condensate steadily changes from horizontal to vertical polarization going through a low DOP regime. We stress that the data in Fig. 4(c) is obtained at a different position of the cavity sample compared to Figs. 1-3 which leads to finite $S_{1}$ at zero PL ellipticity $(a=b)$ even though $\Omega_{\text {trap }}=0$. This is because of local birefringence in the cavity mirrors giving rise to an additional static in-plane magnetic field $\boldsymbol{\Omega}_{\mathrm{bir}}(\mathbf{r})$. Therefore, one needs to account for a net field $\boldsymbol{\Omega}_{\text {net }}=\boldsymbol{\Omega}_{\text {bir }}(\mathbf{r})+\boldsymbol{\Omega}_{\text {trap }}$ orientating the condensate pseudospin. The point of low DOP in Fig. 4(c) corresponds then to near cancellation between the local birefringence and TE-TM splitting $\boldsymbol{\Omega}_{\text {net }} \approx 0$.

\section{COUPLED CONDENSATES}

\section{A. Experimental observations}

Networks of coupled polariton condensates ${ }^{50,57}$ can be seen as an attractive platform to study synchronization phenomena between nonlinear optical elements like microlaser arrays ${ }^{58}$, and to investigate the behaviour of complex nonequilibrium many-body polariton systems and excitations in non-Hermitian lattices ${ }^{24,59-61}$. Inspired by these studies, we create two identical, spatially separated, elliptical optical traps utilizing two SLMs resulting in the formation of two coupled condensates [Fig. 5].

There are two intriguing possibilities in our interacting system. Firstly, we can adjust the fine structure of each optical trap through its major axis angle. Second, the interaction strength between different condensates depends on their relative major axis angle since the trap anisotropy allows polaritons to escape faster along its 

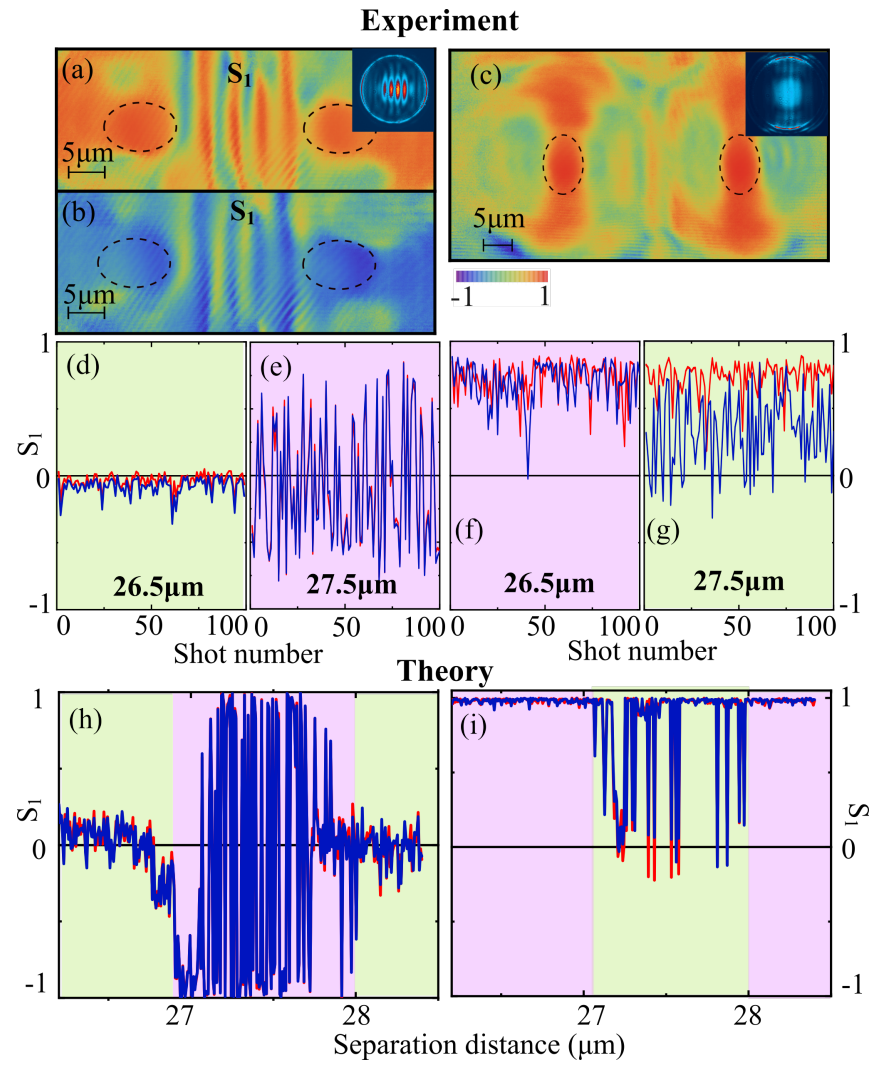

Figure 5. Spatially resolved $S_{1}$ of coupled condensates oriented horizontally and separated by $27.5 \mu \mathrm{m}$ for two different quasi-CW $50 \mu$ s excitation shots (a,b), showing the formation of either horizontal (a) or vertical polarization (b) in both condensates. (c) Spatially resolved $S_{1}$ from one realization of the two coupled condensates orientated vertically at $26.5 \mu \mathrm{m}$. Insets in (a) and (c) depict the corresponding $k$-space PL. (d,e) 100 time-integrated realizations (shots) of the $S_{1}$ for the left (blue) and right (red) condensate in the horizontal-horizontal major axis configuration and $(\mathrm{f}, \mathrm{g})$ in the vertical-vertical configuration. The experimental data is taken at $\approx 1.8 P_{t h}$. Distance dependence of the $S_{1}$ from simulations corresponding to (h) horizontal-horizontal and (i) vertical-vertical major axes configuration. Each datapoint represents one shot (time averaged). Green and purple backgrounds in (d)-(i) illustrate regions of similar behaviour between experiment and theory.

major axis [see Fig. 1(b)]. This can also be evidenced in Fig. 1(e) as high concentration of co-aligned PL in momentum space. This leads to stronger coupling when the traps major axes are orientated longitudinally to the coupling direction, and weaker when orientated transverse (estimated as 3 times weaker from energy resolved spatial PL). The difference in the coupling strength can be evidenced from the different visibility in the momen- tum space interference fringes (implying synchronization) shown in the insets of Figs. 5(a) and 5(c).

Here, we investigate the polarization of the coupled condensates for each of the quasi-CW $50 \mu$ s excitation shots using Wollaston prism and detection camera. Polarization resolving 100 shots, we observe distinct regimes depending on the condensates separation distance and their mutual orientation. For strongly coupled horizontally elongated traps separated by $26.5 \mu \mathrm{m}$ we observe approximately zero DOP in each shot [Fig. 5(d)] for each condensate (blue and red curves, respectively). On the other hand, at a $27.5 \mu \mathrm{m}$ distance we now observe a strong $S_{1}$ component stochastically flipping from shot to shot [Fig. 5(e)] with small $S_{2,3}$ (see Sec. S9 in SI for supplemental data on the other Stokes components). Both condensates in this case adopt the same linear polarization, randomly changing between horizontal [Fig. 5(a)] and vertical [Fig. 5(b)] from shot to shot. Interestingly, the polarization of the condensates are almost perfectly correlated (Pearson correlation coefficient $\rho=0.99)$ which implies that they are strongly coupled. Such random linear polarization flipping suggests the presence of bistability in our system ${ }^{7}$ triggered by the spatial coupling mechanism.

On the other hand, for the weakly coupled (vertically elongated) traps [see Fig. 5(c)] we observe qualitatively different behaviour. Choosing again the same distances, we now see at $26.6 \mu \mathrm{m}$ strong positive $S_{1}$ component in each condensate [Fig. 5(f)] like one would expect for an isolated vertical trap. However, interestingly, at 27.5 $\mu \mathrm{m}$ we observe appearance of semi-depolarized behaviour [Fig. 5(g)] evidenced through stronger polarization fluctuations from shot-to-shot. In both cases, due to the weaker spatial coupling, the condensates are no longer strongly correlated in their $S_{1}$ components with $\rho=0.5$ and $\rho=0.26$ for the two studied distances, respectively. We point out the different average $S_{1}$ values between the left and the right condensate in Fig. $5(\mathrm{~g})$ which we attribute to the position-dependent random birefringence $\boldsymbol{\Omega}_{\text {bir }}(\mathbf{r})$.

\section{B. Theory of coupled condensates}

We reproduce our experimental observations through mean field simulations on time-delay coupled spinor polariton condensates. Here, we model the dynamics of each condensate spinor $\Psi=\left(\psi_{+}, \psi_{-}\right)^{T}$ using the generalized (driven-dissipative) Gross-Pitaevskii equation, coupled to a semiclassical rate equation describing a reservoir of low-momentum excitons $\mathbf{X}=\left(X_{+}, X_{-}\right)^{T}$ which scatter into the condensate ${ }^{62}$. The indexes (1) and (2) denote the left and right condensate respectively. 


$$
\begin{aligned}
i \frac{d \psi_{ \pm}^{(1,2)}}{d t} & =\left[\omega_{0}+\alpha\left|\psi_{ \pm}^{(1,2)}\right|^{2}+g X_{ \pm}^{(1,2)}+i \frac{R X_{ \pm}^{(1,2)}-\Gamma}{2}\right] \psi_{ \pm}^{(1,2)}+(\epsilon+i \gamma) \psi_{\mp}^{(1,2)}+J \psi_{ \pm}^{(2,1)}(t-\tau)+\mathcal{J} \psi_{\mp}^{(2,1)}(t-\tau), \\
\frac{d X_{ \pm}^{(1,2)}}{d t} & =P-\left(\Gamma_{R}+R\left|\psi_{ \pm}^{(1,2)}\right|^{2}\right) X_{ \pm}^{(1,2)}+\Gamma_{s}\left(X_{\mp}^{(1,2)}-X_{ \pm}^{(1,2)}\right) .
\end{aligned}
$$

Here, $\alpha$ and $g$ are the repulsive (defocusing) interaction constants describing the polariton-polariton and polariton-reservoir blueshift, $R$ governs the stimulated scattering rate from the reservoir into the condensate, $\Gamma$ and $\Gamma_{R}$ are the polariton and exciton reservoir decay rates, $\Gamma_{s}$ is the rate of spin relaxation within the reservoirs, $\epsilon$ and $\gamma$ describe the complex-valued fine structure (taken here between horizontal and vertical polarized modes), and $P$ denotes the nonresonant pumping power. For a linearly polarized CW pump we can safely neglect the polarization- and time-dependence of any highmomentum exciton reservoirs describing excitons that are too energetic to scatter into the condensate ${ }^{63}$. We have also introduced the condensate intrinsic energy $\omega_{0}$ since a suitable rotating reference frame cannot be chosen for time-delayed coupled oscillators.

The inter-condensate-coupling, denoted with $J, \mathcal{J} \in \mathbb{C}$, is sometimes referred to as ballistic coupling which was recently described through time-delay coupled equations of motion ${ }^{57}$. Energetic polaritons escape from each trap and undergo finite-time free-space propagation before they reach their neighboring condensate. Such coupling becomes qualitatively different from evanescent coupling (e.g., tunneling between trapped Bose-Einstein condensates) when the propagation time $\tau$ of particles between condensates is comparable to their intrinsic frequencies. As was previously demonstrated in $^{57}$, the strength of the coupling $J$ depends on the separation distance $d$ between the condensates,

$$
J(d)=J_{0}\left|H_{0}^{(1)}\left(k_{c} d\right)\right|,
$$

where $H_{0}^{(1)}$ is the zeroth order Hankel function of the first kind, $J_{0} \in \mathbb{C}$ quantifies the non-Hermitian coupling strength dictated by the overlap of the condensates over the optical trap region, and $k_{c}$ is the complex wavevector of the polaritons propagating outside the optical trap,

$$
k_{c}=k_{c}^{(0)}+i \frac{\Gamma m}{2 \hbar k_{c}^{(0)}} .
$$

From experiment, we have estimated $k_{c}^{(0)} \approx 1.35 \mu \mathrm{m}^{-1}$. This value corresponds to the radius of the outer momentum space PL ring shown in Fig. 1(e). The imaginary term in Eq. (7) describes the additional attenuation of polaritons due to their finite lifetime and $m$ is the polariton effective mass. We also account for coupling between the spins of the two condensates due to the TE-TM splitting which is captured with the parameter $\mathcal{J}$. The time delay parameter is approximated from the polariton phase velocity which gives,

$$
\tau=\frac{2 d m}{\hbar k_{c}^{(0)}} .
$$

The results from simulations for both strongly and weakly coupled traps are presented in Figs. 5(h) and 5(i), respectively. We scan the distance $d$ between the condensates (each datapoint is one shot using random initial conditions) and observe periodic appearance of dramatically different dynamics (green and purple backgrounds) similar to the phase-flip transitions recently reported in $^{57}$.

For the strongly coupled traps, Fig. 5(h) indeed shows that - depending on distance - one can retrieve very low time-averaged polarization which we will refer to as the unstable regime. There, complex interplay of spincoupling and ballistic coupling result in chaotic dynamics depicted in Fig. 6(a) and 6(b). Between $27 \mu \mathrm{m}$ and $28 \mu \mathrm{m}$ a high amplitude random $S_{1}$ component builds up from shot-to-shot due to the condensate spinors suddenly synchronizing as depicted in Figs. 6(c) and 6(d). These results are in qualitative agreement with those seen in experiment in Figs. 5(d) and 5(e). The parameters of the simulation are: $\epsilon=-0.01 \mathrm{ps}^{-1} ; \gamma=\epsilon / 2 ; \Gamma=0.25 \mathrm{ps}^{-1}$; $\Gamma_{R}=\Gamma / 4 ; \Gamma_{s}=\Gamma_{R} / 2 ; \alpha=0.15 \epsilon ; R=0.05 \epsilon ; \omega_{0}=5.5 \Gamma ;$ $g=\alpha ; m=0.3 \mathrm{meV} \mathrm{ps}^{2} \mu \mathrm{m}^{-2} ; J_{0}=0.67 e^{1.8 i} \mathrm{ps}^{-1}$; and $\mathcal{J}=0.2 J$.

For the weakly coupled traps we again obtain qualitative agreement between theory [Fig. 5(i)] and experiment [Figs. 5(f) and 5(g)]. As mentioned in Sec. IV A, the weak coupling between condensates is estimated three times smaller than the strong coupling. This is implemented into the simulation by simply scaling $J_{0} \rightarrow J_{0} / 3$. Now, strong $S_{1} \approx 1$ regimes appear [purple background in Fig. 5(i)] interleaved by regimes of semi-depolarized behaviour [green background in Fig. 5(i)].

It is beyond the scope of the current study to provide an exhaustive analysis on the dynamics of the coupled condensates. We end this section by providing an insightful wider scan in distance and power in Figs. 6(e) and 6(f) for the case of strongly coupled condensates. Each pixel represents one realization of the time averaged $S_{1,2}$ from the left condensate (the right condensate shows nearly identical behavior). The threshold power is derived for a single condensate as $P_{\mathrm{th}}=(\Gamma-2|\gamma|) \Gamma_{R} / R$. At high powers we observe the condensates stabilizing into the ground state (horizontal) polarization [red colors 


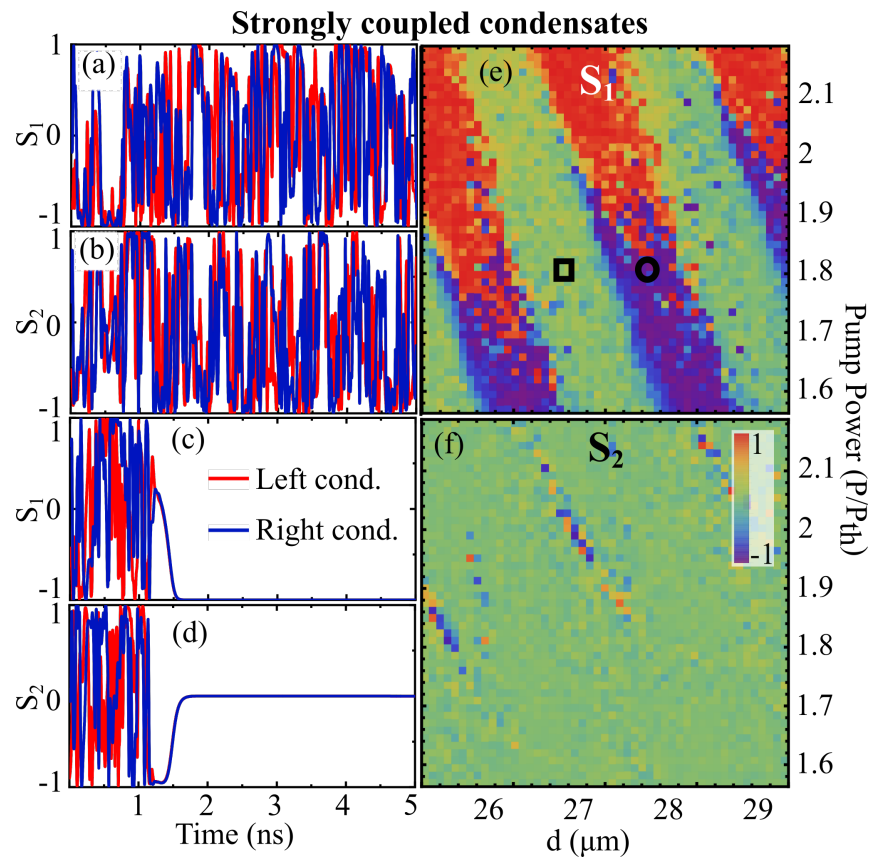

Figure 6. Simulated time evolution of the $S_{1}(t)$ and $S_{2}(t)$ for a single realization of the condensate at the (a),(b) the black square and (c),(d) the black circle in Fig. 6(e), corresponding to the unstable and stable regimes, respectively. Red and blue color correspond to left and right condensate respectively. In panels (e) and (f) we show heatmaps of the numerically obtained time averaged Stokes parameters $S_{1,2}$ of one condensate from random initial conditions and varying pump power and distance.

in Figs. 6(e,f)] whereas at low power we retrieve stable excited state (vertical) polarization condensation [blue colors in Figs. 6(e,f)]. Between these regions we observe an intermediate region (seen as a mixture of blue and red datapoints) where the ground and excited state condensates are both stable and the random initial condition determines the final state. This region corresponds to the experimental observation in Figs. 5 (a),(b) and (e). We observe slanted regions of complete depolarization (green color) corresponding to the unstable regime. Supplemental numerical data for completeness are presented in Sec. S8 in the SI.

\section{CONCLUSIONS}

We have investigated the steady state polarization dynamics of a polariton condensate in an elliptically shaped trapping potential created through optical nonresonant linearly polarized excitation. We have demonstrated that the condensate polarization is determined by the lifted spin-degeneracy of the trap levels due to the trap's geometric ellipticity and TE-TM splitting. The condensate always forms with a linear polarization that follows the minor axis of the trap ellipse. By rotating the excitation profile, we rotate the condensate linear polarization around the equatorial plane of the Poincaré sphere. We then extended our system to a pair of coupled condensates, revealing rich physics of synchronization and desynchronization by tuning the condensate coupling strength through the trap anisotropy and/or spatial separation.

We believe that our results could provide one of the missing pieces of the puzzle for creating all-optical spin circuits in spinoptronic applications. Moreover, it paves the way towards coherent light sources with on-demand switchable linear polarization and studies on extended systems of interacting nonlinear optical elements with anisotropic coupling strength and adjustable fine structure. Here, the response of the SLM limits the polarization rotation speed. However, other approaches of modifying the laser profile with much higher speed, for example interference of two Laguerre-Gaussian laser beams ${ }^{64,65}$ with different wavelength, could result in a generation of sources of coherent light with rotating polarization at gigahertz operation rates.

The data presented in this paper are openly available from the University of Southampton repository.

\section{ACKNOWLEDGEMENTS}

The authors acknowledge the support of the UK's Engineering and Physical Sciences Research Council (grant EP/M025330/1 on Hybrid Polaritonics) and by RFBR according to the research projects No. 20-02-00919. H.S. acknowledges the Icelandic Research Fund, grant No. 217631-051.
* I. GnusovIvan.Gnusov@skoltech.ru

$\dagger$ H. Sigurdsson h.sigurdsson@soton.ac.uk

1 A. Kavokin, J. J. Baumberg, G. Malpuech, and F. P. Laussy, Microcavities (OUP Oxford, 2007).

2 J. Kasprzak, M. Richard, S. Kundermann, A. Baas, P. Jeambrun, J. M. J. Keeling, F. M. Marchetti, M. H. Szymańska, R. André, J. L. Staehli, V. Savona, P. B. Littlewood, B. Deveaud, and L. S. Dang, Bose-Einstein condensation of exciton polaritons., Nature 443, 409 (2006).

${ }^{3}$ I. A. Shelykh, A. V. Kavokin, Y. G. Rubo, T. C. H. Liew, and G. Malpuech, Polariton polarization-sensitive phenom- ena in planar semiconductor microcavities, Semiconductor Science and Technology 25, 013001 (2009).

4 T. Liew, I. Shelykh, and G. Malpuech, Polaritonic devices, Physica E: Low-dimensional Systems and Nanostructures 43, 1543 (2011).

${ }^{5}$ L. Pickup, K. Kalinin, A. Askitopoulos, Z. Hatzopoulos, P. Savvidis, N. Berloff, and P. Lagoudakis, Optical Bistability under Nonresonant Excitation in Spinor Polariton Condensates, Physical Review Letters 120, 225301 (2018).

6 Y. del Valle-Inclan Redondo, H. Sigurdsson, H. Ohadi, I. A. Shelykh, Y. G. Rubo, Z. Hatzopoulos, P. G. Savvidis, 
and J. J. Baumberg, Observation of inversion, hysteresis, and collapse of spin in optically trapped polariton condensates, Physical Review B 99, 165311 (2019).

7 H. Sigurdsson, Hysteresis in linearly polarized nonresonantly driven exciton-polariton condensates, Physical Review Research 2, 023323 (2020).

8 T. K. Paraïso, M. Wouters, Y. Léger, F. Morier-Genoud, and B. Deveaud-Plédran, Multistability of a coherent spin ensemble in a semiconductor microcavity, Nature Materials 9, 655 (2010).

9 A. Amo, T. C. H. Liew, C. Adrados, R. Houdré, E. Giacobino, A. V. Kavokin, and A. Bramati, Exciton-polariton spin switches, Nature Photonics 4, 361 (2010).

10 R. Cerna, Y. Léger, T. K. Paraïso, M. Wouters, F. MorierGenoud, M. T. Portella-Oberli, and B. Deveaud, Ultrafast tristable spin memory of a coherent polariton gas, Nature Communications 4, 2008 (2013).

11 C. Leyder, M. Romanelli, J. P. Karr, E. Giacobino, T. C. H. Liew, M. M. Glazov, A. V. Kavokin, G. Malpuech, and A. Bramati, Observation of the optical spin Hall effect, Nature Physics 3, 628 (2007).

12 R. Hivet, H. Flayac, D. D. Solnyshkov, D. Tanese, T. Boulier, D. Andreoli, E. Giacobino, J. Bloch, A. Bramati, G. Malpuech, and A. Amo, Half-solitons in a polariton quantum fluid behave like magnetic monopoles, Nature Physics 8, 724 (2012).

13 M. Sich, L. E. Tapia-Rodriguez, H. Sigurdsson, P. M. Walker, E. Clarke, I. A. Shelykh, B. Royall, E. S. Sedov, A. V. Kavokin, D. V. Skryabin, M. S. Skolnick, and D. N. Krizhanovskii, Spin domains in one-dimensional conservative polariton solitons, ACS Photonics 5, 5095 (2018).

14 K. G. Lagoudakis, T. Ostatnický, A. V. Kavokin, Y. G. Rubo, R. André, and B. Deveaud-Plédran, Observation of half-quantum vortices in an exciton-polariton condensate, Science 326, 974 (2009).

15 S. Donati, L. Dominici, G. Dagvadorj, D. Ballarini, M. De Giorgi, A. Bramati, G. Gigli, Y. G. Rubo, M. H. Szymańska, and D. Sanvitto, Twist of generalized skyrmions and spin vortices in a polariton superfluid, Proceedings of the National Academy of Sciences 113, 14926 (2016).

16 S. Dufferwiel, F. Li, E. Cancellieri, L. Giriunas, A. A. P. Trichet, D. M. Whittaker, P. M. Walker, F. Fras, E. Clarke, J. M. Smith, M. S. Skolnick, and D. N. Krizhanovskii, Spin textures of exciton-polaritons in a tunable microcavity with large te-tm splitting, Phys. Rev. Lett. 115, 246401 (2015).

17 H. Ohadi, A. Dreismann, Y. Rubo, F. Pinsker, Y. del Valle-Inclan Redondo, S. Tsintzos, Z. Hatzopoulos, P. Savvidis, and J. Baumberg, Spontaneous Spin Bifurcations and Ferromagnetic Phase Transitions in a Spinor ExcitonPolariton Condensate, Physical Review X 5, 031002 (2015).

18 O. Bleu, D. D. Solnyshkov, and G. Malpuech, Interacting quantum fluid in a polariton chern insulator, Phys. Rev. B 93, 085438 (2016).

19 H. Sigurdsson, Y. S. Krivosenko, I. V. Iorsh, I. A. Shelykh, and A. V. Nalitov, Spontaneous topological transitions in a honeycomb lattice of exciton-polariton condensates due to spin bifurcations, Phys. Rev. B 100, 235444 (2019).

20 T. Gao, C. Antón, T. C. H. Liew, M. D. Martín, Z. Hatzopoulos, L. Viña, P. S. Eldridge, and P. G. Savvidis, Spin selective filtering of polariton condensate flow, Applied Physics Letters 107, 011106 (2015).
21 A. Dreismann, H. Ohadi, Y. del Valle-Inclan Redondo, R. Balili, Y. G. Rubo, S. I. Tsintzos, G. Deligeorgis, Z. Hatzopoulos, P. G. Savvidis, and J. J. Baumberg, A sub-femtojoule electrical spin-switch based on optically trapped polariton condensates, Nature Materials 15, 1074 (2016).

22 A. Askitopoulos, A. V. Nalitov, E. S. Sedov, L. Pickup, E. D. Cherotchenko, Z. Hatzopoulos, P. G. Savvidis, A. V. Kavokin, and P. G. Lagoudakis, All-optical quantum fluid spin beam splitter, Physical Review B 97, 235303 (2018).

23 E. S. Sedov, Y. G. Rubo, and A. V. Kavokin, Polariton polarization rectifier, Light: Science \& Applications 8, 1 (2019).

24 S. Mandal, R. Banerjee, E. A. Ostrovskaya, and T. C. H. Liew, Nonreciprocal transport of exciton polaritons in a non-hermitian chain, Physical Review Letters 125, 123902 (2020).

25 J. M. Ostermann and R. Michalzik, Polarization control of vcsels, in VCSELs: Fundamentals, Technology and Applications of Vertical-Cavity Surface-Emitting Lasers, edited by R. Michalzik (Springer Berlin Heidelberg, Berlin, Heidelberg, 2013) pp. 147-179.

26 M. Lindemann, G. Xu, T. Pusch, R. Michalzik, M. Hofmann, I. Žutić, and N. Gerhardt, Ultrafast spin-lasers, Nature 568, 1 (2019).

27 M. Drong, T. Fördös, H. Jaffrès, J. Peřina, K. Postava, P. Ciompa, J. Pištora, and H.-J. Drouhin, Spin-vcsels with local optical anisotropies: Toward terahertz polarization modulation, Phys. Rev. Applied 15, 014041 (2021).

28 J. Hu, L.-s. Li, W. Yang, L. Manna, L.-w. Wang, and A. P. Alivisatos, Linearly polarized emission from colloidal semiconductor quantum rods, Science 292, 2060 (2001).

29 X. Wang, A. M. Jones, K. L. Seyler, V. Tran, Y. Jia, H. Zhao, H. Wang, L. Yang, X. Xu, and F. Xia, Highly anisotropic and robust excitons in monolayer black phosphorus, Nature Nanotechnology 10, 517 (2015).

30 A. Lundskog, C.-W. Hsu, K. Fredrik Karlsson, S. Amloy, D. Nilsson, U. Forsberg, P. Olof Holtz, and E. Janzén, Direct generation of linearly polarized photon emission with designated orientations from site-controlled ingan quantum dots, Light: Science \& Applications 3, e139 (2014).

31 D. N. Krizhanovskii, D. Sanvitto, I. A. Shelykh, M. M. Glazov, G. Malpuech, D. D. Solnyshkov, A. Kavokin, S. Ceccarelli, M. S. Skolnick, and J. S. Roberts, Rotation of the plane of polarization of light in a semiconductor microcavity, Phys. Rev. B 73, 073303 (2006).

32 I. Gnusov, H. Sigurdsson, S. Baryshev, T. Ermatov, A. Askitopoulos, and P. G. Lagoudakis, Optical orientation, polarization pinning, and depolarization dynamics in optically confined polariton condensates, Physical Review B 102, 125419 (2020).

33 H. Ohadi, E. Kammann, T. C. H. Liew, K. G. Lagoudakis, A. V. Kavokin, and P. G. Lagoudakis, Spontaneous Symmetry Breaking in a Polariton and Photon Laser, Physical Review Letters 109, 016404 (2012).

34 J. J. Baumberg, A. V. Kavokin, S. Christopoulos, A. J. D. Grundy, R. Butté, G. Christmann, D. D. Solnyshkov, G. Malpuech, G. Baldassarri Höger von Högersthal, E. Feltin, J.-F. Carlin, and N. Grandjean, Spontaneous Polarization Buildup in a Room-Temperature Polariton Laser, Physical Review Letters 101, 136409 (2008).

35 M. D. Martín, D. Ballarini, A. Amo, Ł. Kłopotowski, L. Viña, A. V. Kavokin, and R. André, Striking dynamics of ii-vi microcavity polaritons after linearly polarized 
excitation, physica status solidi (c) 2, 3880 (2005).

36 Ł. Kłopotowski, M. Martín, A. Amo, L. Viña, I. Shelykh, M. Glazov, G. Malpuech, A. Kavokin, and R. André, Optical anisotropy and pinning of the linear polarization of light in semiconductor microcavities, Solid State Communications 139, 511 (2006).

37 J. Kasprzak, R. André, L. S. Dang, I. A. Shelykh, A. V. Kavokin, Y. G. Rubo, K. V. Kavokin, and G. Malpuech, Build up and pinning of linear polarization in the Bose condensates of exciton polaritons, Physical Review B 75, 045326 (2007).

${ }^{38}$ R. Balili, V. Hartwell, D. Snoke, L. Pfeiffer, and K. West, Bose-Einstein Condensation of Microcavity Polaritons in a Trap, Science 316, 1007 (2007).

39 D. Read, T. C. H. Liew, Y. G. Rubo, and A. V. Kavokin, Stochastic polarization formation in excitonpolariton bose-einstein condensates, Physical Review B 80, 195309 (2009).

40 S. Gerhardt, M. Deppisch, S. Betzold, T. H. Harder, T. C. H. Liew, A. Predojević, S. Höfling, and C. Schneider, Polarization-dependent light-matter coupling and highly indistinguishable resonant fluorescence photons from quantum dot-micropillar cavities with elliptical cross section, Physical Review B 100, 115305 (2019).

41 M. Klaas, O. A. Egorov, T. C. H. Liew, A. Nalitov, V. Marković, H. Suchomel, T. H. Harder, S. Betzold, E. A. Ostrovskaya, A. Kavokin, S. Klembt, S. Höfling, and C. Schneider, Nonresonant spin selection methods and polarization control in exciton-polariton condensates, Physical Review B 99, 115303 (2019).

${ }^{42}$ L. Xiang, X. Zhang, J. Zhang, Y. Huang, W. Hofmann, Y. Ning, and L. Wang, Vcsel mode and polarization control by an elliptic dielectric mode filter, Appl. Opt. 57, 8467 (2018).

43 B. Gayral, J. M. Gérard, B. Legrand, E. Costard, and V. Thierry-Mieg, Optical study of gaas/alas pillar microcavities with elliptical cross section, Applied Physics Letters 72, 1421 (1998).

${ }^{44}$ K. D. Choquette and R. E. Leibenguth, Control of verticalcavity laser polarization with anisotropic transverse cavity geometries, IEEE Photonics Technology Letters 6, 40 (1994).

45 T. Pusch, E. La Tona, M. Lindemann, N. C. Gerhardt, M. R. Hofmann, and R. Michalzik, Monolithic verticalcavity surface-emitting laser with thermally tunable birefringence, Applied Physics Letters 110, 151106 (2017).

46 J. Plumhof, T. Stöferle, L. Mai, U. Scherf, and R. Mahrt, Room-temperature bose-einstein condensation of cavity exciton-polaritons in a polymer, Nature materials $\mathbf{1 3}$ (2013).

47 G. Panzarini, L. C. Andreani, A. Armitage, D. Baxter, M. S. Skolnick, V. N. Astratov, J. S. Roberts, A. V. Kavokin, M. R. Vladimirova, and M. A. Kaliteevski, Excitonlight coupling in single and coupled semiconductor microcavities: Polariton dispersion and polarization splitting, Phys. Rev. B 59, 5082 (1999).

48 P. Cilibrizzi, A. Askitopoulos, M. Silva, F. Bastiman, E. Clarke, J. M. Zajac, W. Langbein, and P. G. Lagoudakis, Polariton condensation in a straincompensated planar microcavity with InGaAs quantum wells, Applied Physics Letters 105, 191118 (2014).

49 E. Wertz, L. Ferrier, D. D. Solnyshkov, R. Johne, D. Sanvitto, A. Lemaître, I. Sagnes, R. Grousson, A. V. Kavokin, P. Senellart, G. Malpuech, and J. Bloch, Spontaneous for- mation and optical manipulation of extended polariton condensates, Nature Physics 6, 860 (2010).

50 G. Tosi, G. Christmann, N. G. Berloff, P. Tsotsis, T. Gao, Z. Hatzopoulos, P. G. Savvidis, and J. J. Baumberg, Sculpting oscillators with light within a nonlinear quantum fluid, Nature Physics 8, 190 (2012).

51 A. Askitopoulos, H. Ohadi, A. V. Kavokin, Z. Hatzopoulos, P. G. Savvidis, and P. G. Lagoudakis, Polariton condensation in an optically induced two-dimensional potential, Physical Review B 88, 041308 (2013).

52 I. I. Ryzhov, V. O. Kozlov, N. S. Kuznetsov, I. Y. Chestnov, A. V. Kavokin, A. Tzimis, Z. Hatzopoulos, P. G. Savvidis, G. G. Kozlov, and V. S. Zapasskii, Spin noise signatures of the self-induced larmor precession, Phys. Rev. Research 2, 022064 (2020).

53 C. Redlich, B. Lingnau, S. Holzinger, E. Schlottmann, S. Kreinberg, C. Schneider, M. Kamp, S. Höfling, J. Wolters, S. Reitzenstein, and K. Lüdge, Mode-switching induced super-thermal bunching in quantum-dot microlasers, New Journal of Physics 18, 063011 (2016).

54 M. Maragkou, C. E. Richards, T. Ostatnický, A. J. D. Grundy, J. Zajac, M. Hugues, W. Langbein, and P. G. Lagoudakis, Optical analogue of the spin hall effect in a photonic cavity, Opt. Lett. 36, 1095 (2011).

${ }^{55}$ L. A. Smirnov, D. A. Smirnova, E. A. Ostrovskaya, and Y. S. Kivshar, Dynamics and stability of dark solitons in exciton-polariton condensates, Phys. Rev. B 89, 235310 (2014).

56 M. Maragkou, A. J. D. Grundy, E. Wertz, A. Lemaître, I. Sagnes, P. Senellart, J. Bloch, and P. G. Lagoudakis, Spontaneous nonground state polariton condensation in pillar microcavities, Phys. Rev. B 81, 081307 (2010).

57 J. Töpfer, H. Sigurdsson, L. Pickup, and P. Lagoudakis, Time-delay polaritonics, Communications Physics 3, 2 (2020).

58 X. Qiao, B. Midya, Z. Gao, Z. Zhang, H. Zhao, T. Wu, J. Yim, R. Agarwal, N. M. Litchinitser, and L. Feng, Higher-dimensional supersymmetric microlaser arrays, Science 372, 403 (2021).

${ }^{59}$ H. Ohadi, A. Ramsay, H. Sigurdsson, Y. del ValleInclan Redondo, S. Tsintzos, Z. Hatzopoulos, T. Liew, I. Shelykh, Y. Rubo, P. Savvidis, and J. Baumberg, Spin Order and Phase Transitions in Chains of Polariton Condensates, Physical Review Letters 119, 067401 (2017).

60 J. D. Töpfer, I. Chatzopoulos, H. Sigurdsson, T. Cookson, Y. G. Rubo, and P. G. Lagoudakis, Engineering spatial coherence in lattices of polariton condensates, Optica 8, 106 (2021).

61 M. Pieczarka, E. Estrecho, S. Ghosh, M. Wurdack, M. Steger, D. W. Snoke, K. West, L. N. Pfeiffer, T. . C. H. Liew, A. G. Truscott, and E. A. Ostrovskaya, Topological phase transition in an all-optical exciton-polariton lattice, arXiv e-prints , arXiv:2102.01262 (2021), arXiv:2102.01262 [physics.optics].

62 M. Wouters and I. Carusotto, Excitations in a nonequilibrium Bose-Einstein condensate of exciton polaritons, Phys. Rev. Lett. 99, 140402 (2007).

63 C. Antón, S. Morina, T. Gao, P. S. Eldridge, T. C. H. Liew, M. D. Martín, Z. Hatzopoulos, P. G. Savvidis, I. A. Shelykh, and L. Viña, Optical control of spin textures in quasi-one-dimensional polariton condensates, Phys. Rev. B 91, 075305 (2015).

64 S. Franke-Arnold, J. Leach, M. J. Padgett, V. E. Lembessis, D. Ellinas, A. J. Wright, J. M. Girkin, P. Öhberg, 
and A. S. Arnold, Optical ferris wheel for ultracold atoms, Opt. Express 15, 8619 (2007).

${ }^{65}$ Y. V. Kartashov and D. A. Zezyulin, Rotating patterns in polariton condensates in ring-shaped potentials under a bichromatic pump, Opt. Lett. 44, 4805 (2019). 